\title{
Comparison of effectiveness and outcomes among different thrombectomy techniques in acute basilar artery occlusion: a dual-center experience
}

\author{
Andre Monteiro, MD, ${ }^{1,2}$ Gustavo M. Cortez, MD, ${ }^{3}$ Muhammad Waqas, MBBS, ${ }^{1,2}$ Hamid H. Rai, MD, ${ }^{1,2}$ \\ Ammad A. Baig, MD,1,2 Rimal H. Dossani, MD, 1,2 Justin M. Cappuzzo, MD, ${ }^{1,2}$ \\ Faisal Almayman, MD, ${ }^{1,2}$ Amin Aghaebrahim, MD, ${ }^{3}$ Jason M. Davies, MD, PhD, ,2,4-6 \\ Eric Sauvageau, MD, ${ }^{3}$ Kenneth V. Snyder, MD, PhD, ${ }^{1,2,5,6}$ Ricardo A. Hanel, MD, PhD, ${ }^{3}$ \\ Elad I. Levy, MD, MBA, ${ }^{1,2,5-7}$ and Adnan H. Siddiqui, MD, PhD ${ }^{1,2,5-7}$
}

Departments of ${ }^{1}$ Neurosurgery, ${ }^{4}$ Bioinformatics, and ${ }^{7}$ Radiology, Jacobs School of Medicine and Biomedical Sciences, and ${ }^{5}$ Canon Stroke and Vascular Research Center, University at Buffalo; ${ }^{2}$ Department of Neurosurgery, Gates Vascular Institute at Kaleida Health; ${ }^{6}$ Jacobs Institute, Buffalo, New York; and ${ }^{3}$ Department of Cerebrovascular and Endovascular Surgery, Baptist Neurological Institute and Lyerly Neurosurgery, Jacksonville, Florida

OBJECTIVE Acute basilar artery occlusion (BAO) is a rare large-vessel occlusion associated with high morbidity and mortality. Modern thrombectomy with stent retrievers and large-bore aspiration catheters is highly effective in achieving recanalization, but a direct comparison of different techniques for acute BAO has not been performed. Therefore, the authors sought to compare the technical effectiveness and clinical outcomes of stent retriever-assisted aspiration (SRA), aspiration alone (AA), and a stent retriever with or without manual aspiration (SR) for treatment of patients presenting with acute $\mathrm{BAO}$ and to evaluate predictors of clinical outcome in their cohort.

METHODS A retrospective analysis of databases of large-vessel occlusion treated with endovascular intervention at two US endovascular neurosurgery centers was conducted. Patients $\geq 18$ years of age with acute BAO treated between January 2013 and December 2020 with stent retrievers or large-bore aspiration catheters were included in the study. Demographic information, procedural details, angiographic results, and clinical outcomes were extracted for analysis.

RESULTS Eighty-three patients (median age 67 years [IQR 58-76 years]) were included in the study; 33 patients (39.8\%) were female. The median admission National Institutes of Health Stroke Scale (NIHSS) score was 16 (IQR 10-21). Intravenous alteplase was administered to 26 patients (31.3\%). The median time from symptom onset to groin or wrist puncture was 256 minutes (IQR 157.5-363.0 minutes). Overall, successful recanalization was achieved in 74 patients (89.2\%). The SRA technique had a significantly higher rate of modified first-pass effect (mFPE; $55 \%$ vs $31.8 \%, p=$ 0.032 ) but not true first-pass effect (FPE; $45 \%$ vs $34.9 \%, p=0.346$ ) than non-SRA techniques. Good outcome (modified Rankin Scale [mRS] score 0-2) was not significantly different among the three techniques. Poor outcome (mRS score 3-6) was associated with a higher median admission NIHSS score (12.5 vs 19, $p=0.007)$, a higher rate of adjunctive therapy usage ( $9 \%$ vs $0 \%, p<0.001)$, and a higher rate of intraprocedural complications $(10.7 \%$ vs $14.5 \%, p=0.006)$. The admission NIHSS score significantly predicted good outcome (OR 0.98, 95\% CI 0.97-0.099; $p=0.032$ ). Incomplete recanalization after thrombectomy significantly predicted mortality (OR 1.68, 95\% Cl 1.18-2.39; $p=0.005)$.

CONCLUSIONS The evaluated techniques resulted in high recanalization rates. The SRA technique was associated with a higher rate of mFPE than AA and SR, but the clinical outcomes were similar. A lower admission NIHSS score predicted a better prognosis for patients, whereas incomplete recanalization after thrombectomy predicted mortality.

https://thejns.org/doi/abs/10.3171/2021.4.FOCUS21114

KEYWORDS acute basilar artery occlusion; stroke; thrombectomy; technique; aspiration; stent retriever; effectiveness; clinical outcomes

ABBREVIATIONS $A A=$ aspiration alone; $B A O=$ basilar artery occlusion; $F P E=$ first-pass effect; $I A=$ intraarterial; $I V=$ intravenous; $m F P E=$ modified FPE; $m R S=$ modified Rankin Scale; $\mathrm{mTICI}=$ modified Thrombolysis in Cerebral Infarction; NIHSS = National Institutes of Health Stroke Scale; sICH = symptomatic intracranial hemorrhage; $\mathrm{SR}=$ stent retriever alone or in combination with manual aspiration; SRA = stent retriever-assisted aspiration; tPA = tissue plasminogen activator

SUBMITTED February 25, 2021. ACCEPTED April 6, 2021.

INCLUDE WHEN CITING DOI: 10.3171/2021.4.FOCUS21114. 
A CUTE basilar artery occlusion (BAO) is a rare but deadly form of large-vessel occlusion leading to the infarction of several eloquent and critical structures within the surroundings of the brainstem. In the past, severe disability or death was reported to occur in almost $90 \%$ of patients treated with antithrombotic medications or pharmacological thrombolysis, mostly due to low rates of recanalization., ${ }^{1,2}$ In 2009, the Basilar Artery International Cooperation Study (BASICS) compared different therapies for acute $\mathrm{BAO}$ and found no significant benefit of intraarterial (IA) modalities over intravenous (IV) alteplase. ${ }^{3}$ Since publication of the BASICS, endovascular options have improved remarkably with the usage of improved catheters and stent retrievers. Currently, most of the evidence supporting the use of modern thrombectomy devices comes from observational studies. In part, this is due to the drastic superiority in recanalization rates obtained with modern devices, raising ethics concerns about randomization of patients with acute BAO. In fact, the Acute Basilar Artery Occlusion: Endovascular Interventions vs Standard Medical Treatment (BEST) trial ended prematurely due to loss of equipoise, which generated a high cross-over rate from the conservative arm to the endovascular arm. ${ }^{4}$ The BASICS randomized prospective trial was recently completed and the results should soon be available, while the Basilar Artery Occlusion Chinese Endovascular (BAOCHE) trial (https://clinicaltrials.gov/ct2/show/NCT02737189) is still enrolling patients. ${ }^{5}$ However, comparison of different thrombectomy techniques, such as stent retriever-assisted aspiration (SRA), aspiration alone (AA), and the use of stent retrievers alone or in combination with manual aspiration (SR), in acute $\mathrm{BAO}$ has not yet been performed.

We performed a retrospective analysis of the experience with the treatment of acute $\mathrm{BAO}$ at two neurosurgery centers. Our primary objective was to compare the technical effectiveness among current thrombectomy techniques using modern stent retrievers and large-bore aspiration catheters. As a secondary objective, we evaluated potential predictors of clinical outcomes in our cohort.

\section{Methods}

\section{Population and Data}

This was a retrospective study using the prospectively collected databases of patients with large-vessel occlusion treated with endovascular intervention between January 2013 and December 2020. The study was approved by the institutional review board at each center. Inclusion criteria were 1 ) $\geq 18$ years of age, 2) thrombus located at or extending into the basilar artery, and 3) treatment with a stent retriever or a large-bore aspiration catheter. The variables extracted were age, sex, comorbidities (i.e., hypertension, diabetes, hyperlipidemia, and atrial fibrillation), National Institutes of Health Stroke Scale (NIHSS) score at admission, location of the occlusion, presence of a tandem lesion, presence of underlying vertebrobasilar atherosclerotic disease, use of IV recombinant tissue plasminogen activator (tPA; alteplase), time from symptom onset to groin or wrist puncture and recanalization, modality and device of the endovascular thrombectomy, number of passes made in an attempt to obtain recanalization, use of adjunctive therapies (e.g., IA thrombolytics, glycoprotein IIb/IIIa inhibitors, angioplasty), final modified Thrombolysis in Cerebral Infarction (mTICI) grade, intraprocedural complications, occurrence of symptomatic postprocedure hemorrhage, and modified Rankin Scale (mRS) score 90 days after the procedure.

\section{Definitions}

Clinical outcomes at 90 days were dichotomized into good outcome (mRS score $\leq 2)$ and poor outcome (mRS score $>2$ ). The location of the occlusion along the basilar artery was classified into proximal, middle, or distal based on the segments determined by the branching point of the anterior inferior cerebellar artery and superior cerebellar artery. ${ }^{6}$ Recanalization was classified as successful (mTICI grade $2 b-3$ ) or incomplete (mTICI grade $<2 b$ ). Futile recanalization was defined as an mTICI grade $2 b-3$ (successful recanalization) that did not translate into a good outcome. Modified first-pass effect (mFPE) was based on a previous definition as an mTICI grade $2 \mathrm{~b}-3$ within a single pass of the technique. ${ }^{7}$ First-pass effect (FPE) was defined as an mTICI grade $2 \mathrm{c}-3$ within a single pass. ${ }^{7}$ The definition of symptomatic intracranial hemorrhage (sICH) followed the Heidelberg Bleeding Classification system. ${ }^{8}$

\section{Endovascular Thrombectomy}

Devices and techniques were used at the discretion of each operator. The SRA technique employed a stent retriever in combination with an aspiration catheter connected to an automated suction system. The AA technique involved the use of an aspiration catheter alone as a first attempt to retrieve the clot. The SR technique was a third option, involving use of a stent retriever alone or in combination with manual aspiration. Whenever one technique failed, the decision to perform more passes in an attempt to achieve recanalization or the switch to another technique was made at the operator's discretion.

The choice of access (i.e., radial vs femoral) was based on anatomical factors and operator preference. The side of the dominant vertebral artery and the aortic arch type were important in influencing the choice of access. Radial access was generally preferred for right vertebral artery dominant cases. For radial access, a 6F sheath was placed in the right radial artery. A 96- $\mathrm{cm} 6 \mathrm{~F}$ Benchmark catheter (Penumbra) was advanced over an angled Berenstein catheter (Merit Medical) and positioned in the distal $\mathrm{V}_{2}$ segment. A $125-\mathrm{cm} 5 \mathrm{~F}$ Sofia catheter (MicroVention) was then advanced over a 0.025 -inch or a 0.027 -inch microcatheter and a 0.014-inch microwire. The lesion was crossed with a J-shaped 0.014-inch microwire for SR and SRA. For AA-first cases, the aspiration catheter was advanced to the clot interface for aspiration.

For femoral cases, an $8 \mathrm{~F}$ sheath was placed. An $8 \mathrm{~F} \mathrm{Neu}-$ ron Max guide catheter (Penumbra) was advanced over a Berenstein catheter and positioned in the $\mathrm{V}_{2}$ segment. A 0.068-inch or 0.071-inch aspiration catheter was advanced over a 0.025 -inch Velocity microcatheter (Penumbra) over a 0.014-inch microwire. For SR or SRA-first cases, the lesion was crossed with a J-shaped 0.014-inch microwire, and the stent retriever device was deployed. The device 
was kept deployed for up to 3 minutes prior to retrieval. For SRA cases, the aspiration catheter was brought up to the clot interface before active pump aspiration.

\section{Statistical Analysis}

Continuous variables were presented as means and SD or as medians and IQR, depending on data normality. Categorical variables were presented as proportions. Continuous variables were compared using the Student t-test or the Mann-Whitney U-test. Categorical variables were compared using the chi-square or Fisher exact test. Multivariate logistic regression models were constructed using relevant factors while accounting for significance on univariate analysis. A 2 -sided $p$ value $<0.05$ was considered significant in all analyses. Missing data were handled with nonparametric missing value imputation using a random forest algorithm (missForest, The R Foundation). All statistical analyses were performed using R software (https:// www.R-project.org/).

\section{Results}

Eighty-three patients (median age 67 years [IQR 58-76 years]) were included in the study; 33 (39.8\%) were female. The most common comorbidities were hypertension (69.9\%) and hyperlipidemia (36.1\%). The median NIHSS score at admission was 16 (IQR 10-21). Intravenous alteplase was administered to 26 patients (31.3\%). The median time from symptom onset to groin or wrist puncture was 256 minutes (IQR 157.5-363.0 minutes), and from puncture to recanalization it was 42 minutes (IQR 31-60 minutes). The occluded segment of the basilar artery was proximal in 21 patients $(25.3 \%)$, midbasilar in 26 patients $(31.3 \%)$, and distal in 36 patients $(43.4 \%)$. SRA was the primary technique in 40 patients $(48.2 \%)$, AA in 23 patients (27.7\%), and SR in 20 patients (24.1\%). Adjunctive therapy was needed in 5 patients $(6 \%)$, of whom 2 patients were found to have severe flow-limiting stenosis after thrombectomy and required angioplasty, and 2 patients required IA injection of a glycoprotein IIb/IIIa inhibitor (eptifibatide) due to persistent reocclusion after a few passes. In the fifth patient, the microwire could not be advanced across the occlusion. Aspiration was then attempted during several minutes without success. Finally, a decision was made to inject IA alteplase through the microcatheter, still resulting in an mTICI grade of 0 . Intraprocedural complications occurred in 11 patients (13.2\%). Successful recanalization was achieved in 74 patients (89.2\%). Postprocedure sICH was seen in 7 patients (8.4\%). These data, as well as a breakdown by each mRS score, are detailed in Table 1 . The rate of good outcome at 90 days postprocedure was $33.6 \%$. Twenty-five patients (30.1\%) died within 90 days.

\section{Univariate Analysis}

Technique, Recanalization, and Outcomes

Overall, mFPE was achieved in 36 patients (43.4\%) and FPE in 33 patients $(39.7 \%)$. The rate of $\mathrm{mFPE}$ did not significantly differ among SRA (55\%), AA (36.8\%), and SR $(30 \%)(\mathrm{p}=0.130)$. The rate of FPE was also not significantly different between SRA (45\%), AA (34.8\%), and SR $(35 \%)(\mathrm{p}=0.642)$. The rate of good outcome did not sig-
TABLE 1. Baseline characteristics, procedural information, and clinical and angiographic outcomes of the study cohort

\begin{tabular}{|c|c|}
\hline Variable & Value $(n=83)$ \\
\hline Median age, yrs & $67(58-76)$ \\
\hline Female sex & $33(39.8)$ \\
\hline Hypertension & $58(69.9)$ \\
\hline Diabetes & $25(30.1)$ \\
\hline Atrial fibrillation & $12(14.5)$ \\
\hline Hyperlipidemia & $30(36.1)$ \\
\hline Median admission NIHSS score & $16(10-21)$ \\
\hline \multicolumn{2}{|l|}{ Occlusion site } \\
\hline Proximal & $21(25.3)$ \\
\hline Middle & $26(31.3)$ \\
\hline Distal & $36(43.4)$ \\
\hline Underlying vertebrobasilar atherosclerotic disease & $24(28.9)$ \\
\hline IV tPA & $26(31.3)$ \\
\hline Median onset to puncture, mins & $256(157.5-363.0)$ \\
\hline Mean puncture to recanalization, mins & $49.41[28.41]$ \\
\hline Median puncture to recanalization, mins & $42(31-60)$ \\
\hline \multicolumn{2}{|l|}{ Primary thrombectomy technique } \\
\hline SRA & $40(48.2)$ \\
\hline AA & $23(27.7)$ \\
\hline SR & $20(24.1)$ \\
\hline \multicolumn{2}{|l|}{ No. of passes } \\
\hline 1 & $43(51.8)$ \\
\hline 2 & $20(24.1)$ \\
\hline 3 & $12(14.5)$ \\
\hline 4 & $4(4.8)$ \\
\hline 5 & $3(3.6)$ \\
\hline 6 & $1(1.2)$ \\
\hline \multicolumn{2}{|l|}{ Adjunctive therapy } \\
\hline IA thrombolytic & $1(1.2)$ \\
\hline Glycoprotein Ilb/llla inhibitor & $2(2.4)$ \\
\hline Angioplasty & $2(2.4)$ \\
\hline $\mathrm{mTICl}$ grade $2 b-3$ & $74(89.2)$ \\
\hline \multicolumn{2}{|l|}{ Intraprocedural complications } \\
\hline Distal embolization & $4(4.8)$ \\
\hline Reocclusion & $1(1.2)$ \\
\hline Dissection & $4(4.8)$ \\
\hline Vasospasm & $2(2.4)$ \\
\hline Postprocedure sICH & $7(8.4)$ \\
\hline \multicolumn{2}{|l|}{ 90-day mRS score } \\
\hline 0 & $7(8.4)$ \\
\hline 1 & $14(16.8)$ \\
\hline 2 & $7(8.4)$ \\
\hline 3 & $11(13.3)$ \\
\hline 4 & $11(13.3)$ \\
\hline 5 & $8(9.6)$ \\
\hline 6 & $25(30.1)$ \\
\hline
\end{tabular}

Values represent the number of patients (\%) unless stated otherwise. Median values are presented as the median (IQR). Mean values are presented as the mean [SD]. 
TABLE 2. Comparison of patients with good versus poor outcome

\begin{tabular}{|c|c|c|c|}
\hline Variable & $\begin{array}{c}\text { Good Outcome } \\
(m R S \text { score } 0-2)(n=28)\end{array}$ & $\begin{array}{c}\text { Poor Outcome } \\
(m R S \text { score } 3-6)(n=55)\end{array}$ & p Value \\
\hline Median age, yrs & $66(56.75-73.25)$ & $68(59.5-79.5)$ & 0.140 \\
\hline Female sex & $8(28.6)$ & $25(45.5)$ & 0.160 \\
\hline Hypertension & $21(75)$ & $37(67.3)$ & 0.614 \\
\hline Diabetes & $7(25)$ & $18(32.7)$ & 0.614 \\
\hline Atrial fibrillation & $3(10.7)$ & $9(16.4)$ & 0.742 \\
\hline Hyperlipidemia & $13(46.4)$ & $17(30.9)$ & 0.227 \\
\hline Median admission NIHSS score & $12.5(8.25-16)$ & $19(10-23)$ & 0.007 \\
\hline Occlusion site & & & 0.748 \\
\hline Proximal & $7(25)$ & $14(25.5)$ & \\
\hline Middle & $8(28.6)$ & $18(32.7)$ & \\
\hline Distal & $13(46.4)$ & $23(41.8)$ & \\
\hline Underlying vertebrobasilar atherosclerotic disease & $6(21.4)$ & $18(32.7)$ & 0.318 \\
\hline IV tPA & $12(42.9)$ & $14(25.5)$ & 0.138 \\
\hline Median onset to puncture, mins & $250.5(157-492.8)$ & $257(156-359)$ & 0.277 \\
\hline Median puncture to recanalization, mins & $32.00(23.5-50.5)$ & $49.74(35.5-65)$ & 0.173 \\
\hline Mean no. of passes & $1.92[1.4]$ & $1.82[1.1]$ & 0.952 \\
\hline Adjunctive therapies & & & $<0.001$ \\
\hline IA thrombolytic & $0(0)$ & $1(1.8)$ & \\
\hline Glycoprotein Ilb/Illa inhibitor & $0(0)$ & $2(3.6)$ & \\
\hline Angioplasty & $0(0)$ & $2(3.6)$ & \\
\hline $\mathrm{mTICl}$ grade $2 b-3$ & $27(96.4)$ & $47(85.5)$ & 0.261 \\
\hline Intraprocedural complications & & & 0.006 \\
\hline Distal embolization & $0(0)$ & $4(7.3)$ & \\
\hline Reocclusion & $0(0)$ & $1(1.8)$ & \\
\hline Dissection & $2(7.1)$ & $2(3.6)$ & \\
\hline Vasospasm & $1(3.6)$ & $1(1.8)$ & \\
\hline Postprocedure sICH & $0(0)$ & $7(12.7)$ & 0.089 \\
\hline
\end{tabular}

Values represent the number of patients (\%) unless stated otherwise. Median values are presented as the median (IQR). Mean values are presented as the mean [SD]. Boldface type indicates statistical significance.

nificantly differ between patients treated primarily with SRA $(32.5 \%)$, AA $(39.1 \%)$, or SR $(30 \%)(\mathrm{p}=0.797)$.

In dichotomized analysis, SRA was significantly associated with a higher rate of $\mathrm{mFPE}(55 \%$ vs $31.8 \%, \mathrm{p}=0.032)$ but not FPE (45\% vs $34.9 \%, \mathrm{p}=0.346)$ in comparison with non-SRA techniques, whereas AA versus non-AA (mFPE, $\mathrm{p}=0.358 ; \mathrm{FPE}, \mathrm{p}=0.566)$ and SR versus non-SR (mFPE, $p=0.183 ;$ FPE, $p=0.617$ ) had no significant associations. The rate of good outcome was not significantly different between SRA and non-SRA $(\mathrm{p}=0.818)$, AA and non-AA $(p=0.519)$, or $S R$ and non-SR $(p=0.594)$.

In an analysis comparing stent retriever-based techniques (SRA and SR) with AA, there were no significant differences in the rates of mFPE $(46.7 \%$ vs $34.8 \%$, $\mathrm{p}=$ 0.328 ), FPE (41.7\% vs $34.8 \%$; $\mathrm{p}=0.566$ ), and good outcome $(31.7 \%$ vs $39.1 \%, \mathrm{p}=0.519)$.

Overall, futile recanalization was observed in 47 patients (56.6\%). Among the 15 patient procedures that did not achieve mFPE primarily with AA, 5 achieved successful recanalization on the second or third pass after switching to SRA, 2 after switching to SR, and 7 with subsequent
AA attempts. Only 1 patient retained an mTICI grade of 0 after many aspiration attempts and use of adjunctive IA alteplase. Of note, patients treated primarily with SRA or SR whose procedures did not achieve mFPE were not switched to a different technique in subsequent passes.

\section{Moderate Versus Poor Outcomes}

There was no significant difference between good and poor outcome groups regarding age, comorbidities, presence of tandem occlusion or underlying vertebrobasilar atherosclerotic disease, use of IV alteplase, and time to groin or wrist puncture. Poor outcome was associated with a higher median NIHSS score (12.5 vs 19, $\mathrm{p}=0.007)$, a higher rate of adjunctive therapy usage (10\% vs $0 \%$, p < 0.001 ), and a higher rate of intraprocedural complications $(10.7 \%$ vs $14.5 \%, \mathrm{p}=0.006)$. These results are summarized in Table 2.

\section{Multivariate Analysis}

Predictors of Good Outcome

A logistic regression model was performed and in- 
cluded age, NIHSS score at admission, time from onset of symptoms to puncture, occlusion site, technique, and mFPE. Only the admission NIHSS score was found to significantly predict good outcome (OR $0.98,95 \%$ CI $0.97-$ $0.99 ; \mathrm{p}=0.032$ ). These results are seen in Table 3 .

\section{Predictors of Mortality}

A logistic regression model was performed and included age, NIHSS score at admission, time from onset of symptoms to puncture, occlusion site, underlying vertebrobasilar atherosclerotic disease, incomplete reperfusion, postprocedure complications, and postprocedure sICH. The only significant predictor of mortality identified was incomplete recanalization (OR 1.68, 95\% CI 1.18-2.39; p $=0.005)$. These results are summarized in Table 3 .

\section{Discussion}

In our experience, the treatment of acute $\mathrm{BAO}$ with mechanical thrombectomy using modern devices resulted in a high rate of successful recanalization $(89.2 \%$ ) and an acceptable rate of good outcome (33.6\%). The SRA technique demonstrated better technical performance regarding $\mathrm{mFPE}$ over AA and SR, but no difference in good clinical outcomes was found between the three techniques. In univariate analysis, poor outcome was associated with a more severe stroke presentation, a higher rate of periprocedural complications, and a greater need for adjunctive therapies. Furthermore, the only predictors of good and poor outcome were lower admission NIHSS score and incomplete recanalization, respectively.

Analyses of different mechanical thrombectomy techniques have been performed mostly in the anterior circulation, while comparative data in the posterior circulation is sparse. AA was shown to be noninferior in outcomes and to have similar technical efficacy and safety with cost advantages compared with stent retrievers in the randomized comparison of direct aspiration versus stent retriever as a first approach (COMPASS) trial. ${ }^{9}$ However, the SRA technique has not been previously compared with AA or $\mathrm{SR}$, in neither the anterior nor the posterior circulation. ${ }^{2}$ The decision of which technique to use as the primary approach depends on operator experience and judgment about the characteristics of a clot. Usually, a thrombus that is thought to be embolic in nature has a good chance of retrieval with AA. More extensive clots or those associated with underlying atherosclerosis often do not respond well to aspiration alone; in this situation, more robust and aggressive techniques such as SRA may be performed primarily. In fact, a subanalysis of the COMPASS trial demonstrated that clot characteristics such as perviousness have a marked influence on the success of AA but not that of stent retriever thrombectomy..$^{10}$ The specific characteristics of basilar artery clots have not been extensively studied, representing a good direction for future research. Except for the minor differences discussed, all three techniques were effective in our study but did not influence the outcomes of patients.

The procedural success in our cohort is in line with that in previous studies evaluating modern devices in the treatment of acute $\mathrm{BAO}$, reporting recanalization rates be-
TABLE 3. Multivariate analysis of moderate outcome and mortality

\begin{tabular}{|c|c|c|c|}
\hline Covariate & OR & $95 \% \mathrm{Cl}$ & $\mathrm{p}$ Value \\
\hline \multicolumn{4}{|l|}{ Good outcome (mRS score 0-2) } \\
\hline Age & 0.99 & $0.98-1$ & 0.380 \\
\hline Admission NIHSS score & 0.98 & $0.97-0.99$ & 0.032 \\
\hline $\begin{array}{l}\text { Time from symptom onset to } \\
\text { puncture }\end{array}$ & 1 & $0.99-1$ & 0.4678 \\
\hline Proximal occlusion & 1.23 & $0.66-2.28$ & 0.5014 \\
\hline Midbasilar occlusion & 1.13 & $0.62-2.07$ & 0.6803 \\
\hline Distal occlusion & 1.19 & $0.66-2.16$ & 0.5549 \\
\hline mFPE & 1.10 & $0.88-1.36$ & 0.3873 \\
\hline SRA & 1.70 & $0.70-4.10$ & 0.2414 \\
\hline AA & 1.80 & $0.77-4.19$ & 0.1734 \\
\hline Stent retriever & 1.88 & $0.74-4.75$ & 0.1829 \\
\hline \multicolumn{4}{|l|}{ Mortality (mRS score 6) } \\
\hline Age & 1 & $0.99-1.01$ & 0.236 \\
\hline Admission NIHSS score & 1 & $0.99-1.01$ & 0.250 \\
\hline $\begin{array}{l}\text { Time from symptom onset to } \\
\text { puncture }\end{array}$ & 1 & $0.99-1$ & 0.588 \\
\hline Proximal occlusion & 0.66 & $0.38-1.15$ & 0.155 \\
\hline Midbasilar occlusion & 0.76 & $0.43-1.32$ & 0.333 \\
\hline Distal occlusion & 0.78 & $0.45-1.36$ & 0.395 \\
\hline $\begin{array}{l}\text { Underlying vertebrobasilar } \\
\text { atherosclerotic disease }\end{array}$ & 1.17 & $0.93-1.46$ & 0.174 \\
\hline Incomplete recanalization & 1.68 & $1.18-2.39$ & 0.005 \\
\hline Intraprocedural complications & 1.26 & $0.94-1.70$ & 0.124 \\
\hline Postprocedure sICH & 1.21 & $0.85-1.74$ & 0.283 \\
\hline
\end{tabular}

tween 64\% and 100\%. ${ }^{11-21}$ Optimal recanalization was previously demonstrated to impact mortality and disability in these patients in a previous meta-analysis. ${ }^{22}$ Our findings correspond with such results, as incomplete recanalization was the only predictor of mortality in our cohort. Although the rates of futile recanalization reported in the literature remain considerable, ours included (56.6\%), they are actually similar to the rates in anterior circulation thrombectomy. ${ }^{23,24}$ In real-world practice, thrombectomy for anterior circulation large-vessel occlusion is inherently performed under more liberal patient selection because the strict criteria used in clinical trials would preclude a large number of patients from being treated. ${ }^{24}$ In the same way, most operators do not withhold modern thrombectomy from patients with acute BAO, resulting in the futile recanalization rates observed. Despite uncertainty on how future randomized acute BAO studies will affect clinical practice, it is definitely important to continuously investigate parameters that should be considered in treatment decision-making. In a recent systematic review and metaanalysis, Lu et al. found NIHSS score to be the only significant parameter that consistently predicted outcome in studies of acute BAO treated with mechanical thrombectomy. ${ }^{25}$ In our experience, this was also the only baseline characteristic that predicted a good clinical outcome. 
Our study has limitations. This is a retrospective analysis of a small sample size because of the limited population of interest. All procedures were performed by a group of consistent operators with similar endovascular background training, with the advantage of technical and observational consistency but reduced generalizability. Given the aforementioned limitations, the results must be approached with caution.

\section{Conclusions}

Mechanical thrombectomy for the treatment of our patient cohort presenting with acute $\mathrm{BAO}$ resulted in high rates of successful recanalization. SRA had a significantly higher rate of mFPE than AA and SR combined, but clinical outcomes between these techniques were similar. Stroke severity measured by admission NIHSS score could identify patients with a better prognosis, while incomplete recanalization after thrombectomy was an important marker of mortality. Although current techniques are effective and comparable, more studies are needed to investigate the prognostic factors in this patient population.

\section{Acknowledgments}

We thank Junyu Nie, PhD, for statistical analysis and Debra J. Zimmer for editorial assistance.

\section{References}

1. Jadhav AP, Desai SM, Panczykowski DM, et al. Predicting outcomes after acute reperfusion therapy for basilar artery occlusion. Eur J Neurol. 2020;27(11):2176-2184.

2. Munich SA, Vakharia K, Levy EI. Overview of mechanical thrombectomy techniques. Neurosurgery. 2019;85(suppl 1): S60-S67.

3. Schonewille WJ, Wijman CA, Michel P, et al. Treatment and outcomes of acute basilar artery occlusion in the Basilar Artery International Cooperation Study (BASICS): a prospective registry study. Lancet Neurol. 2009;8(8):724-730.

4. Liu X, Dai Q, Ye R, et al. Endovascular treatment versus standard medical treatment for vertebrobasilar artery occlusion (BEST): an open-label, randomised controlled trial. Lancet Neurol. 2020;19(2):115-122.

5. Zi W, Qiu Z, Wu D, et al. Assessment of endovascular treatment for acute basilar artery occlusion via a nationwide prospective registry. JAMA Neurol. 2020;77(5):561-573.

6. Lee WJ, Jung KH, Ryu YJ, et al. Impact of stroke mechanism in acute basilar occlusion with reperfusion therapy. Ann Clin Transl Neurol. 2018;5(3):357-368.

7. Zaidat OO, Castonguay AC, Linfante I, et al. First pass effect: a new measure for stroke thrombectomy devices. Stroke. 2018;49(3):660-666.

8. von Kummer R, Broderick JP, Campbell BC, et al. The Heidelberg Bleeding Classification: classification of bleeding events after ischemic stroke and reperfusion therapy. Stroke. 2015;46(10):2981-2986.

9. Turk AS III, Siddiqui A, Fifi JT, et al. Aspiration thrombectomy versus stent retriever thrombectomy as first-line approach for large vessel occlusion (COMPASS): a multicentre, randomised, open label, blinded outcome, non-inferiority trial. Lancet. 2019;393(10175):998-1008.

10. Mokin M, Waqas M, Fifi J, et al. Clot perviousness is associated with first pass success of aspiration thrombectomy in the COMPASS trial. J Neurointerv Surg. 2021;13(6):509-514.

11. Tran AT, Nguyen HA, Vu DL, et al. Basilar artery thrombec- tomy: assessment of outcome and identification of prognostic factors. Acta Neurol Belg. 2020;120(1):99-105.

12. Andersson T, Kuntze Söderqvist Å, Söderman M, et al. Mechanical thrombectomy as the primary treatment for acute basilar artery occlusion: experience from 5 years of practice. J Neurointerv Surg. 2013;5(3):221-225.

13. Espinosa de Rueda M, Parrilla G, Zamarro J, et al. Treatment of acute vertebrobasilar occlusion using thrombectomy with stent retrievers: initial experience with 18 patients. AJNR Am J Neuroradiol. 2013;34(5):1044-1048.

14. Lutsep HL, Rymer MM, Nesbit GM. Vertebrobasilar revascularization rates and outcomes in the MERCI and multiMERCI trials. J Stroke Cerebrovasc Dis. 2008;17(2):55-57.

15. Pfefferkorn T, Holtmannspötter M, Schmidt C, et al. Drip, ship, and retrieve: cooperative recanalization therapy in acute basilar artery occlusion. Stroke. 2010;41(4):722-726.

16. Mourand I, Machi P, Milhaud D, et al. Mechanical thrombectomy with the Solitaire device in acute basilar artery occlusion. J Neurointerv Surg. 2014;6(3):200-204.

17. Mordasini P, Brekenfeld C, Byrne JV, et al. Technical feasibility and application of mechanical thrombectomy with the Solitaire FR Revascularization Device in acute basilar artery occlusion. AJNR Am J Neuroradiol. 2013;34(1):159-163.

18. Costalat V, Machi P, Lobotesis K, et al. Rescue, combined, and stand-alone thrombectomy in the management of large vessel occlusion stroke using the solitaire device: a prospective 50-patient single-center study: timing, safety, and efficacy. Stroke. 2011;42(7):1929-1935.

19. Delgado Acosta F, Jiménez Gómez E, de Asís Bravo Rodríguez F, et al. Vertebrobasilar recanalization techniques before the placement of stent-retrievers: reopening is not synonymous with good outcome. Article in Spanish. Radiologia. 2014;56(1):44-51.

20. Baek JM, Yoon W, Kim SK, et al. Acute basilar artery occlusion: outcome of mechanical thrombectomy with Solitaire stent within 8 hours of stroke onset. AJNR Am J Neuroradiol. 2014;35(5):989-993.

21. Werner M, Lopez-Rueda A, Zarco F, et al. Mechanical thrombectomy in acute basilar artery occlusion: a safety and efficacy single centre study. Interv Neuroradiol. 2016;22(3): 310-317.

22. Kumar G, Shahripour RB, Alexandrov AV. Recanalization of acute basilar artery occlusion improves outcomes: a metaanalysis. J Neurointerv Surg. 2015;7(12):868-874.

23. Lindsberg PJ, Sairanen T, Nagel S, et al. Recanalization treatments in basilar artery occlusion-systematic analysis. Eur Stroke J. 2016;1(1):41-50.

24. Chen M. Why futile recanalization matters. J Neurointerv Surg. 2020;12(10):925-926.

25. Lu VM, Young CC, Chen SH, et al. Presenting NIHSS predicts 90-day functional outcome after mechanical thrombectomy for basilar artery occlusion: a systematic review and meta-analysis. Clin Neurol Neurosurg. 2020;197:106199.

\section{Disclosures}

Dr. Aghaebrahim: advisory board of iSchemia View. Dr. Davies: research grant from National Center for Advancing Translational Sciences of the National Institutes of Health under award no. KL2TR001413 to the University at Buffalo; consultant for Medtronic; honoraria from Neurotrauma Science; and shareholder/ownership interests in Cerebrotech, RIST Neurovascular. Dr. Snyder: consultant and teacher for Canon Medical Systems, Penumbra, Medtronic, and Jacobs Institute; and co-founder of Neurovascular Diagnostics. Dr. Hanel: consultant for Medtronic, Stryker, Cerenovus, MicroVention, Balt, Phenox, and vizAI; and stock ownership in Neurvana, Elum, Endostream, Three Rivers Medical Inc., Synchron, Deinde, Rist, Cerebrotech Medical Systems Inc., InNeurCo, Serenity, Scientia, BendIT, and Blink TBI. Dr. Levy: 
shareholder/ownership interests in NeXtGen Biologics, RAPID Medical, Claret Medical, Cognition Medical, Imperative Care (formerly the Stroke Project), Rebound Therapeutics, StimMed, and Three Rivers Medical; national principal investigator/steering committees for Medtronic (merged with Covidien Neurovascular) SWIFT Prime and SWIFT Direct trials; honoraria from Medtronic (training and lectures); consultant for Claret Medical, GLG Consulting, Guidepoint Global, Imperative Care, Medtronic, Rebound, and StimMed; advisory board of Stryker (AIS Clinical Advisory Board), NeXtGen Biologics, MEDX, Cognition Medical, Endostream Medical; and site principal investigator for the CONFIDENCE study (MicroVention) and STRATIS Study-Sub I

(Medtronic). Dr. Siddiqui: financial interest/investor/stock options/ ownership: Adona Medical, Amnis Therapeutics (purchased by Boston Scientific October 2017), Blink TBI, Buffalo Technology Partners, Cerebrotech Medical Systems, Cognition Medical, Endostream Medical Ltd., Imperative Care, International Medical Distribution Partners, Neurovascular Diagnostics, Q'Apel Medical, Rebound Therapeutics (purchased by Integra LifeSciences Corp. 2019), Rist Neurovascular, Sense Diagnostics, Serenity Medical, Silk Road Medical, Spinnaker Medical, StimMed, Synchron, Three Rivers Medical, Vastrax, VICIS, and Viseon; consultant/advisory board for Amnis Therapeutics, Boston Scientific, Canon Medical Systems USA, Cerebrotech Medical Systems, Cerenovus, Corindus, Endostream Medical, Imperative Care, Integra LifeSciences, Medtronic, MicroVention, Minnetronix
Neuro, Northwest University-DSMB chair for HEAT Trial, Penumbra, Q'Apel Medical, Rapid Medical, Rebound Therapeutics (purchased by Integra LifeSciences), Serenity Medical, Silk Road Medical, StimMed, Stryker, Three Rivers Medical, VasSol, and W.L. Gore \& Associates; principal investigator/steering committee for Cerenovus (NAPA and ARISE II), Medtronic (SWIFT PRIME and SWIFT DIRECT); MicroVention (FRED and CONFIDENCE), MUSC (POSITIVE), Penumbra 3D Separator, COMPASS, INVEST, and TIGER trials.

\section{Author Contributions}

Conception and design: Monteiro, Waqas. Acquisition of data: Monteiro, Cortez. Analysis and interpretation of data: all authors. Drafting the article: Monteiro, Waqas. Critically revising the article: all authors. Reviewed submitted version of manuscript: all authors.

\section{Correspondence}

Adnan H. Siddiqui: University at Buffalo, NY. asiddiqui@ ubns.com. 